POS PROCEEDINGS

\title{
The silicon vertex telescope of the NA60 experiment
}

\section{André David* for the NA60 Pixel Project}

CERN, Geneva, Switzerland

CFTP, Instituto Superior Técnico, Portugal

E-mail: Andre.Davidecern.ch

M. Floris ${ }^{1}$, G. Usai ${ }^{1}$, K. Banicz ${ }^{2,3}$, M. Keil ${ }^{2,4}$, C. Lourenço ${ }^{2}$, E. Radermacher ${ }^{2}$, R. Shahoyan ${ }^{2,4}$, J. Heuser ${ }^{5}$, H. Ohnishi ${ }^{5}$.

${ }^{1}$ Univ. di Cagliari and INFN, Cagliari, Italy; ${ }^{2}$ CERN, Geneva, Switzerland; ${ }^{3}$ Univ. Heidelberg, Heidelberg, Germany; ${ }^{4}$ CFTP, Instituto Superior Técnico, Lisbon, Portugal; ${ }^{5}$ RIKEN, Wako, Saitama, Japan;

The NA60 experiment at CERN studies the production of open charm and prompt dimuons in collisions of high energy protons or heavy ions with target nuclei. The physics program relies on the precise measurement of the charged particles' positions and momenta at the vertex. Tracking starts already a few centimetres away from the target in a radiation tolerant silicon detector placed in a 2.5 T dipole magnetic field. During several weeks of running in 2003 and 2004, this vertex tracker was exposed to high levels of inhomogeneously distributed radiation. In this paper we describe the construction, operation and performance of this novel, LHC-era detector.

International Europhysics Conference on High Energy Physics

July 21st - 27th 2005

Lisboa, Portugal

${ }^{*}$ Speaker. 


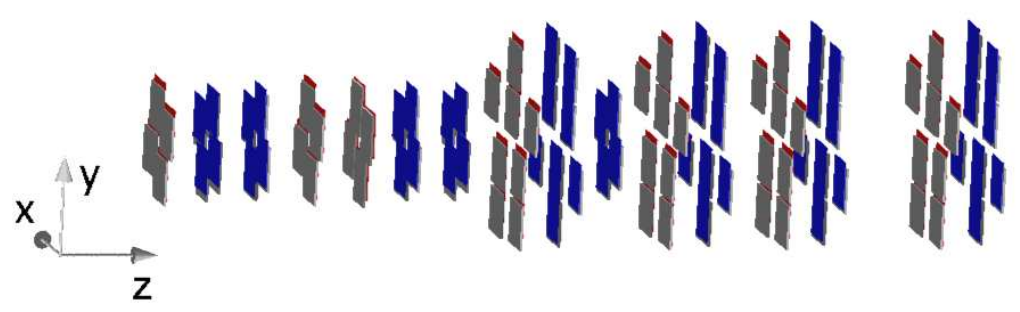

Figure 1: The vertex tracker in the indium run. The beam is in the direction of the $Z$ axis, passing through the holes at the centre of the planes. The first pixel plane was at $z=7 \mathrm{~cm}$, the last at $z=33 \mathrm{~cm}$.

Recent advances in radiation hard silicon devices have made it possible to operate high-precision tracking detectors near the interaction region in heavy-ion experiments for extended periods of time. NA60 built its vertex tracker from silicon pixel chips designed for the ALICE, LHCb and ATLAS experiments, and operated them in a "real-life" physics experiment some four years before these experiments start at CERN's Large Hadron Collider (LHC). The knowledge of event kinematics at this level of precision represents a veritable "quantum leap" in data quality, and signifi cantly widens the range of accessible physics topics, as attested in the most recent results from NA60 [1, 2, 3].

Measurement strategy The coordinates of incident beam particles are measured by a silicon microstrip beam tracker, operated at $130 \mathrm{~K}$ for signifi cantly increased radiation hardness. Interactions take place mostly in the segmented target. The produced charged particles are tracked with the silicon vertex tracker operated inside a $2.5 \mathrm{~T}$ dipole magnetic fi eld. A $5.5 \mathrm{~m}$ thick hadron absorber ensures that only the penetrating high-energy muons make it to the last subdetector system, the muon spectrometer composed of multiwire proportional chambers and scintillator hodoscopes. In addition, a $1.2 \mathrm{~m}$ thick iron wall placed after the last multiwire proportional chamber and before last trigger hodoscope absorbs punch-through hadrons. The requirement of two muons in the muon spectrometer provides a highly selective trigger (one event triggered out of a thousand indium-indium collisions). On the other hand, muons suffer considerable energy loss fluctuations and multiple scattering as they traverse the hadron absorber, which would make meaningful extrapolation back to the vertex region impossible without the help of the vertex tracker. The muons that triggered the event in the muon spectrometer can be selected from the hundreds of vertex tracker tracks by matching them in both coordinate and momentum space. In addition to making it possible to precisely extrapolate the muons to the vertex region, the matching procedure also eliminates a large fraction of the background (pion and kaon decays, interactions outside the target), and improves the accuracy of the momentum measurement.

The vertex tracker For the indium-indium run in 2003, the tracker was built from 96 silicon pixel chips arranged in eight 4-chip planes and eight 8-chip planes (Fig. 1). Each 4-chip plane alone covered the full angular acceptance of the muon spectrometer, while it took two 8-chip planes to do so farther away from the target. Each pixel chip is an assembly of a sensor chip bump-bonded to a frontend readout chip. The ALICE1LHCb readout chip was designed for the ALICE and LHCb experiments by the CERN Microelectronics Group [4]. It was manufactured in a $.25 \mu \mathrm{m}$ CMOS technology and has a radiation tolerant architecture [5]. It has been shown to remain operational 


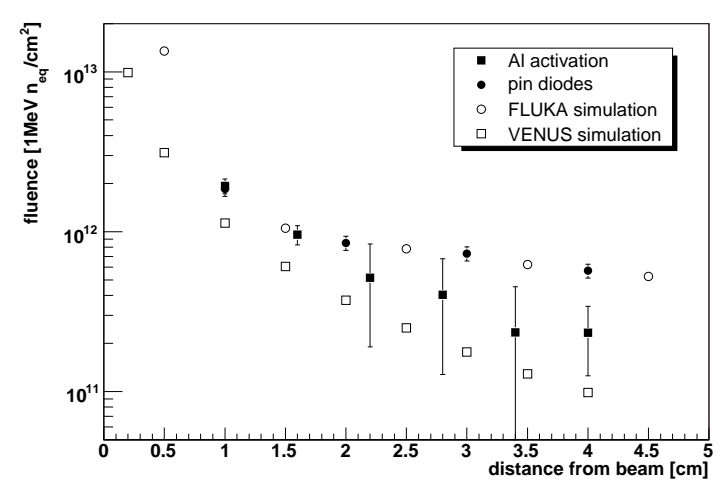

Figure 2: Simulated and measured fluences plotted against the distance from the beam. The FLUKA simulation agrees well with the pin diode measurements, while the VENUS simulation estimates the aluminium activation better.

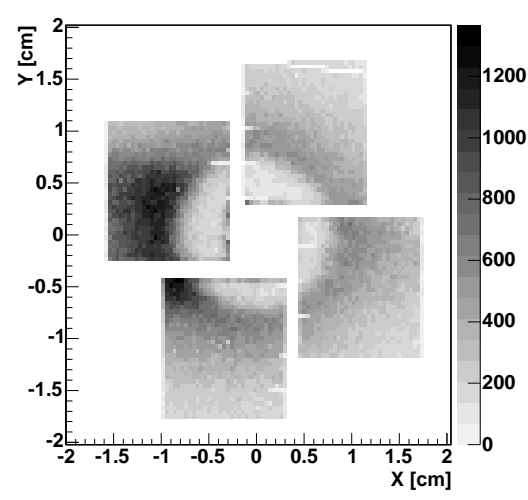

Figure 3: Pixel hits accumulated over a few thousand events. The inefficient central area shows the region where the full depletion voltage is already above the bias voltage of $30 \mathrm{~V}$ being applied.

after an irradiation of $12 \mathrm{Mrad}$ [6]. The sensor and readout chips have a matching $32 \times 256$ matrix of $425 \mu \mathrm{m} \times 50 \mu \mathrm{m}$ pixels. A $25 \mu \mathrm{m}$ diameter solder bump connects each sensor pixel to its counterpart on the readout chip. The chips could be operated safely even at threshold levels below 2000 electrons without incurring appreciable noise. The readout chip is clocked at $10 \mathrm{MHz}$, and its 32 columns are read out in parallel. The four or eight chips on a plane are read out over a serial bus. The sensor chip is a $300 \mu \mathrm{m}$ thick high-resistivity $(15 \mathrm{k} \Omega \mathrm{cm}) n$-type silicon bulk with $p$-type pixel implants. The thickness of the readout chip is $750 \mu \mathrm{m}$.

In 2003 the vertex tracker was operated in a 5-week-long run with a $158 \mathrm{AGeV}$ indium beam incident on a segmented indium target of $16 \%$ interaction probability.

Unlike the muon spectrometer behind the hadron absorber, the vertex tracker is directly exposed to all tracks from all interactions at a close range. The levels and effects of radiation were simulated with the VENUS Monte Carlo generator [9], which took into account primary particles only, and with FLUKA [10], which also included slow secondary and back-scattered particles. During the fi rst three weeks of the run, the fluences were measured with a dosimetric plane placed $40 \mathrm{~cm}$ downstream of the target. It had aluminium rings and silicon pin diodes mounted on it. The fluences were derived from the activation of aluminium in the $\pi+{ }_{13} \mathrm{Al} \rightarrow{ }_{11}^{22} \mathrm{Na}$ process. The cross section of this process drops for low energy pions, making it insensitive to slow particles [7]. In contrast, both high and low energy particles cause bulk damage in pin diodes [8]. For them, the fluences were extracted from the changes in their $I-V$ characteristics. The results and comparison of the simulations and measurements are shown in Fig. 2. From these we infer that the hardest hit pixels were exposed to a fluence of $10^{13} 1 \mathrm{MeVn}_{\mathrm{eq}} \mathrm{cm}^{-2}$ per week.

Radiation damage also changes the effective doping concentration, and the $n$-type bulk eventually becomes effectively $p$-type. After this type inversion takes place, the rectifying junction moves from the $p$-type pixel implants to the $n^{+}$-type back plane. As a consequence, the sensor must be fully depleted in order for the detector to work properly. Also, the depletion voltage decreases with the received fluence until type inversion, and from then on it increases. As pixels closer to the beam receive more fluence, they also have a higher depletion voltage after type inversion. This effect is confi rmed by the measurement shown in Fig. 3. 


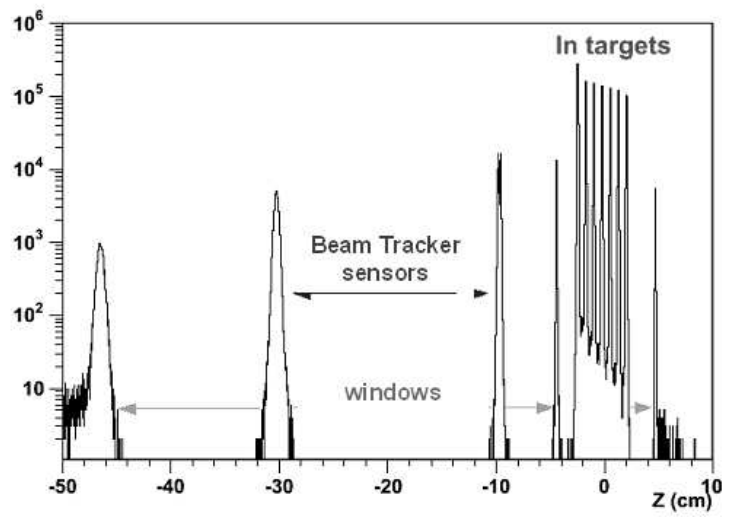

Figure 4: Distribution of the $Z$ coordinates (parallel to the beam) of the reconstructed vertices. Visible are the two stations of the beam tracker between the windows of its vacuum box (at $z=-10 \mathrm{~cm}$ the 2 sensors are discernible as a double peak), and the clearly resolved 7 indium targets between vacuum windows.

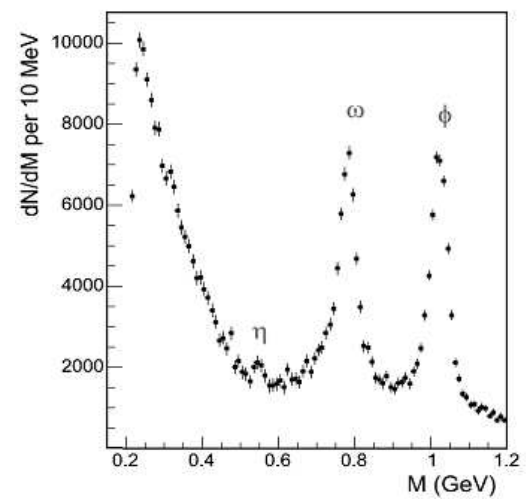

Figure 5: Invariant dimuon mass spectrum obtained by combining both vertex tracker and muon spectrometer information after background subtraction. For the first time in heavy-ion collisions, the $\omega$ and $\phi$ are clearly resolved, and even the two-body $\eta$ decay peak is visible.

Vertexing precision in the direction along the beam is illustrated in Fig. 4. From that distribution we extract a resolution of $\sim 200 \mu \mathrm{m}$. The transverse coordinates of the vertices can be extrapolated from the beam tracker as well as the vertex tracker. From the comparison of these two measurements, and taking into account the beam tracker's known resolution of $20 \mu \mathrm{m}$, we infer a resolution of $10-20 \mu \mathrm{m}$ in the transverse plane, improving with the number of tracks reconstructed in the vertex tracker. The dimuon invariant mass resolution is illustrated in Fig. 5. It would be $70 \mathrm{MeV}$ at the $\omega$ if only the muon spectrometer information were used, and becomes $20 \mathrm{MeV}$ when the vertex tracker measurement is also taken into account.

Closing remarks The successful operation of the vertex tracker of NA60 in 2003 and 2004 has demonstrated that it is now possible to operate a high-speed, high-granularity silicon detector for extended periods of time in the harsh environment of heavy-ion collisions. The authors are grateful to all those who contributed to the success of the NA60 pixel project. Special thanks are due to the pixel groups of the ALICE and ATLAS experiments for their kind support.

\section{References}

[1] R. Shahoyan et al. (NA60 Coll.), Charm and intermediate mass dimuons in In+In collisions, Proc. Quark Matter 2005, to be published in Nucl. Phys. A.

[2] S. Damjanovic et al. (NA60 Coll.), First measurement of the rho spectral function in nuclear collisions, Proc. Quark Matter 2005, [arXiv:nucl-ex/0510044], to be published in Nucl. Phys. A.

[3] R. Arnaldi et al. (NA60 Coll.), Anomalous J/psi suppression in indium-indium collisions at 158 GeV/nucleon, Proc. Quark Matter 2005, to be published in Nucl. Phys. A.

[4] K. Wyllie et al., A pixel readout chip for tracking at ALICE and particle identification at LHCb, Proc. of the Fifth Workshop on Electronics for LHC Experiments, Snowmass, Colorado, 1999.

[5] W. Snoeys et al., Nucl. Instr. and Meth. A 466 (2001) 366.

[6] J.J. van Hunen et al., Irradiation and SPS Beam Tests of the ALICE1LHCb Pixel Chip, CERN-ALI-2001-015, 2001.

[7] Landolt-Börnstein, I/13, Radionuclide Production, Springer-Verlag

[8] M. Moll et al., Nucl. Instr. Meth. Phys. Res. B 186 (2002)

[9] K. Werner, Phys. Rep. 232 (1993) 87.

[10] A. Fassò et al., eConf C0303241, MOMT004 (2003) [arXiv:physics/0306162]. 